\title{
15 Fazit: Wissenschaftsurheberrecht - ein Recht für Nutzungsrechte und Nutzungsfreiheiten
}

Das Urheberrecht, insbesondere das Wissenschaftsurheberecht, hat systematisch ein dogmatisches und ein Problem in seiner Rechtsrealität - und damit auch ein Akzeptanzproblem bei den davon betroffenen Wissenschaftlern. Das erste Problem ist eins der Fundamente, das zweite ein Problem des Hauses, welches auf diesen Fundamenten errichtet wurde und an dem immer noch weiter gewerkelt wird. $\mathrm{Zu}$ den Fundamenten sind zu rechnen den Geist des 19. Jahrhunderts widerspiegelnde Pfeiler wie z. B. die Konzepte von Schöpfung/Schöpfer, Werk, Wissen als Immaterialgut, Monismus (Einheit von persönlichkeits- und vermögensrechtlichen Ansprüchen der Urheber), Eigentums- und Vergütungsansprüche. Wenn - um noch einmal auf die Skepsis von (Dreier/Hilty 2015) zurückzukommen - die Fundamente brüchig geworden sind, kann das Haus nicht länger Bestand haben.

Die Fundamente des Urheberrechts mögen für eine gewisse Zeit im 19. Jahrhundert sinnvoll gewesen sein - so der Schutz des Urhebers, des kreativen Schöpfers vor dem einschränkenden Zensuransinnen einer undemokratischen Obrigkeit im 19. Jahrhundert. Veränderte Rahmenbedingungen - technologische, soziale, politische Entwicklungen, andere Märkte und daraus entwickelte moralische Einstellungen - lassen das Urheberrecht heute mit der Übernahme von Prinzipien aus dem 19. Jahrhundert und daraus abgeleiteten Regulierungen wie aus der Zeit gefallen erscheinen. Das Urheberrecht hält seine Systematik und Dogmatik durch eine Reihe von heute obsolet angenommenen Als-ob-Annahmen, Fiktionen, aufrecht. Diese haben sich in den oben erwähnten Fundamenten verfestigt. Eine Weile kann über das Als-ob Verhalten - daran sei mit Vaihinger erinnert - aus Fiktionen durchaus etwas Nützliches entstehen. Aber sie können auch dysfunktional werden, wenn aus ihnen normative Verpflichtungen mit ungewollten negativen „Nebenfolgen“ entstehen. Das trifft besonders für die Fiktion der immateriellen Objekte zu, die, vor allem für Bildung und Wissenschaft, dysfunktional mit negativen Effekten geworden sind.

(Peukert 2018) hat überzeugend nachgewiesen, dass dem ,immateriellen Objekt“ gar keine ontologische Realität entspricht - was aber, als Sieg des das Urheberrecht konstituierenden Als-ob-Verhaltens, die Juristen nicht daran gehindert hat, diese Fiktion als soziale Realität anzuerkennen und entsprechende rechtlich verbindliche Regelungen für den Umgang damit festzulegen. Wenn etwas zu einem Objekt erklärt wurde (sei es auch „nur“ als ein immaterielles Objekt) und wenn das allgemeine und damit soziale und politische Anerkennung gefunden hat, dann ist es auch gutsfähig. Das Gut „Wissen“ kann kommodifiziert und entsprechend kann mit ihm nach Marktprinzipien gehandelt werden. Das Urheberrecht tut so,

Ә Open Access. (C) 2020 Rainer Kuhlen (c))BY This work is licensed under a Creative Commons Attribution 4.0 License. https://doi.org/10.1515/9783110693447-015 
als ob es mit seinen Regelungen die Interessen der Urheber, der Ersteller von Wissensobjekten, schützt, während es tatsächlich immer mehr die Interessen der Verwerter unterstützt. Wenigen außerhalb der Rechtswissenschaft ist vermutlich bewusst, dass es diese Fiktionen sind, die dafür verantwortlich sind, dass das Haus im Urheberrecht so unkomfortabel für Bildung und Wissenschaft eingerichtet wurde. Die Metapher des Hauses bezieht sich auf die positiven Urheberrechtsgesetze, die sich u. a. aus den erwähnten Konzepten ableiten. Kleine Reparaturen, Instandsetzungen, wie sie im Gesetz immer wieder unternommen werden (zuletzt durch das UrhWissG 2017/18), helfen nicht.

Das derzeit geltende Urheberrecht ist für Bildung und Wissenschaft eher behindernd, in weiten Teilen sogar überflüssig. Politiker und Institutionen wie Parteien, die das Urheberrecht formulieren, haben in den letzten 20 Jahren zu wenig auf die Transformationsprozesse durch die drei Regulierungsinstanzen, Technologie, Markt und Wertewandel und auf die dadurch entstandenen Leitideen für den Umgang mit Wissen und Information reagiert. Dass der deutsche Gesetzgeber, unterstützt auch von vielen Rechtswissenschaftlern, sich immer wieder auf die internationalen, urheberrechtlich relevanten Vorgaben bezogen hat, die ihm keinen weiteren Spielraum böten, kann nicht als Entlastung akzeptiert werden. Zum einen wäre der Spielraum viel größer gewesen, wenn man sich mehr um kreative als um affirmative Hermeneutik bemüht hätte. Auch die internationalen Vorgaben arbeiten mit unbestimmten Rechtsbegriffen, die auslegungsbedürftig und -fähig sind. Sie könnten ganz anders als bislang verstanden werden. Das trifft auch für programmatische Vorgaben wie den Drei-Stufen-Test zu, für den eine andere Interpretation nötig und möglich ist. Ebenso können und sollten manche anderen Begriffe in Schrankenregelungen des Urheberrechts neu verstanden werden. Wir haben das am Beispiel der Bibliotheken gezeigt, bei denen man zu ganz anderen Regulierungen käme, wenn sie nicht mehr als Gebäude, sondern als virtuelle Organisationen begriffen würden.

Vor allem für Bildung und Wissenschaft sind die erwähnten Fundamente nicht tragfähig. Solange aus den alten, dysfunktional gewordenen Konzepten weiter Rechtsvorschriften abgeleitet werden, ist heute im 21. Jahrhundert für Bildung und Wissenschaft vom Urheberrecht wenig zu erwarten. Dem Recht ist es nicht gelungen bzw. der Gesetzgeber hat es auch nicht gewollt, der aus den alten Fundamenten sich ableitenden Kommodifizierung bzw. der daraus folgenden Ökonomisierung und damit der Einbindung des Urheberrechts in ein Handelsrecht Einhalt zu gebieten. Das Urheberrecht ist entsprechend weiter davon ausgegangen, die Informationswirtschaft schützen zu müssen, und hat immer weiter an Schrankenbestimmungen gebastelt, die sich nur durch Ausnahmen von den exklusiven Rechten der Rechtsinhaber rechtfertigen und die an der Praxis und den Bedürfnissen in Bildung und Wissenschaft vorbeigehen. Es hat weiter darauf gesetzt, dass den Verwertern nicht 
nur für die Erstellung ihrer Informationsprodukte eine Vergütung zusteht, sondern auch über die in den Schrankenregelungen geregelten aktuellen Nutzungen.

Die Probleme mit dem Urheberrecht spiegeln sich auch in der Benennung dieses Rechts wider. Ein Recht, das sich letztlich aus dem öffentlichen Interesse an Wissen und Information begründet, sollte nicht auf ein individuelles Recht, auf ein exklusives Recht der Urheber reduziert werden. Das Urheberrecht sollte vom Konzept des Urhebers als individueller Schöpfer befreit werden und damit von dem Recht, über diese „Schöpfungen“ exklusiv verwertend verfügen zu können. Die exklusive Rechtsverfügung des Urhebers - auch das Recht, alle Verwertungsrechte als Nutzungsrechte an kommerzielle Verwerter abgeben zu dürfen - ist die Ursache für die Kommodifizierung und Ökonomisierung auch von öffentlich finanziertem Wissen. Je stärker das Urheberrecht die Position des Urhebers macht, desto stärker, so paradox das klingt, unterstützt es die Interessen der Verwerter. Das ist gerade nicht im Interesse des wissenschaftlichen Urhebers. Das belegen die immer häufiger und stärker werdenden Widerstände aus der Wissenschaft gegen die kommerzielle Aneignung und Ausbeutung ihrer Wissensobjekte vor allem durch das big business großer internationaler Verlagskonsortien. Auch das Wissenschaftsurheberrecht - und diese Einschätzung ist wohl heute keine vernachlässigungsbedürftige Fehlinterpretation - ist immer mehr zu einem Handelsrecht, zu einem Investitionsschutzrecht geworden, zu einer Schutzfunktion des digitalen Kapitalismus der Gegenwart (vgl. Staab 2019). Maximilian Becker spricht von der „Zweckentfremdung des Urheberrechts als zentrales Wirtschaftsrecht des Internets“.638

Viele internationale Förderorganisationen ziehen daraus die Konsequenz und verlangen inzwischen, dass es nicht bei dem exklusiven freien Recht der wissenschaftlichen Autoren bleiben darf, welches den Weg zur verknappenden Verwertung freimacht, sondern dass Publikationen von öffentlich finanzierten Werken frei nach Open-Access-Prinzipien verfügbar sein müssen. In Deutschland, anders als in vielen anderen Ländern, ist es noch für viele Betroffene, auch für Autoren, problematisch, wenn diese freie Verfügbarkeit über eine Mandatierung erzwungen werden soll. Erfolgversprechender wäre es vielleicht, den Weg in Richtung einer umfassenden Open-Access-Transformation dadurch zu befördern, dass das individuelle Recht explizit um ein am Gemeinwohlinteresse orientiertes institutionelles Recht erweitert würde. Diese Position hat sich der deutsche Gesetzgeber bislang nicht angeschlossen, obgleich der Anlass bei der Einführung eines Zweitverwertungsrechts dies nahegelegt hätte.

Ein institutionelles Recht schließt den Schutz der Interessen der „Urheber“ in keiner Weise aus. Auch das Wissenschaftsurheberrecht, wie es hier vorgeschlagen

638 (Becker 2019) Von der Freiheit, rechtswidrig handeln zu können, S. 648. 
wird, garantiert die Persönlichkeitsrechte. Autoren haben weiter das erste, zur Wissenschaftsfreiheit zu rechnende exklusive Recht, ihr produziertes Wissen öffentlich zu machen. Daraus müssen aber keine individuellen exklusiven Verwertungsrechte folgen. Schon gar nicht muss ihnen das Recht zugestanden werden, die im Urheberrecht ihnen zugesicherten Verwertungsrechte vollständig als Nutzungsrechte an kommerzielle Verwerter zu übertragen, ohne dass die sie tragenden und finanzierenden Hochschulen und Forschungseinrichtungen darüber ein Mitspracherecht haben - wie es im Patentrecht der Fall geworden ist. Zur Wissenschaftsfreiheit kann es nicht gehören, alle Nutzungsrechte vollständig an kommerzielle Verwerter übertragen zu dürfen - mit dem Ergebnis, dass die Rechte der Öffentlichkeit (und damit der Wissenschaft) an der Nutzung von Informationsobjekten unbillig eingeschränkt werden.

Nichts muss im Recht so bleiben, wie es ist - also auch nicht die Unterstützung oder Tolerierung der Kommodifizierung von Wissen durch das Urheberrecht. Gegenwärtig artikuliert sich auf vielen Gebieten von Wirtschaft und Gesellschaft der Widerstand gegen die ökonomische Dominanz und den staatlich unterstützten Privatisierungsansprüchen gegenüber materiellen und immateriellen Ressourcen, welche für das Wohlergehen aller Menschen oder sogar für Leben aller Menschen entscheidend sind. Genannt seien hier nur Wasser, Energie, Luft, Klima, Bildung, Gesundheit, Verkehr, Wohnen, Nahrung - und eben auch Wissen und Information. Das Konzept der Commons (Gemein-/Allmendegüter), das sich auf diese unverzichtbaren Ressourcen bezieht, erlebt in den letzten Jahren weltweit eine Renaissance. Für den Umgang mit den letztlich commons-basierten Ressourcen haben sich seit einigen Jahren neue Leitideen entwickelt, die Auswirkungen auf die politischen, sozialen und ökonomischen Strukturen und eben auch auf das Recht haben (werden). Auch diese neuen Leitideen waren anfänglich nur Fiktionen oder sogar nur Träume (wie der zu Beginn in (FN 61) erwähnte Traum von Grötschel). Aber in ihrer institutionellen Verdichtung zu Leitideen haben sie durchaus eine pragmatische, konstruktive handlungsleitende Funktion - auch eine befreiende Wirkung von früheren Fiktionen, die damals auch die pragmatische Leistung der Befreiung von bevormundenden und kontrollierenden Systemen erbrachten, aber heute nicht mehr befreiend wirken.

Für jedermann ersichtlich sind neue Leitideen bezüglich der Ressourcen Luft/ Klima/Energie. Der Zeitgeist ist eindeutig ökologisch bestimmt und wird auch in Politik, Gesellschaft und Wirtschaft akzeptiert und hat für die politischen, sozialen und ökonomischen Strukturen und Verhaltensweisen Konsequenzen. Wirtschaft ohne Ökologie ist nicht mehr denkbar. Ökologie allgemein war nicht das Thema dieser Darstellung. Aber es verfertigt sich in der Öffentlichkeit auch so etwas wie eine Wissensökologie für den Umgang mit immateriellen Objekten (ohne dass diese Benennung schon umfassend Eingang im Sprachgebrauch gefunden 
hat). Wissensökologie, der nachhaltige Umgang mit Wissen und Information, ist das Pendant zur allgemeinen Ökologie für den nachhaltigen Umgang mit materiellen Objekten. ${ }^{639}$ Die hier geübte Kritik an der Kommodifizierung von Wissen und Information und an dem Zögern von Rechtsetzung, der Gesetzgebung und Rechtsprechung ist Teil des gegenwärtigen Unbehagens an bzw. des Protestes gegen die Zerstörung der Ressourcen durch sich verselbständigende ökonomische Interessen.

Das Reden über den Umgang mit Wissen und Information gestaltet sich in der Wissenschaft zunehmend als ein Reden über den offenen, nutzungs- und vergütungsfreien Zugriff auf das öffentlich entstandene und dann publizierte Wissen. Das ist die sich seit etwa 20 Jahren entwickelnde Leitidee. ${ }^{640}$ Darüber wird zunehmend in der Wissenschaft, aber immer mehr auch weltweit bei den politischen Instanzen gesprochen. Es bleibt dann nicht beim Reden. Das sich verfestigende Reden, das, was Peukert in Rückgriff auf Searle einen deklarativen Sprechakt genannt hat, wird zur sozialen Realität. Diese Realität gewinnt Gestalt im Handeln der davon Betroffenen, sich die Nutzungsfreiheit von Wissen und Information zu sichern. Konkretisiert und operationalisierbar wird inzwischen diese sozusagen institutionalisierte Leitidee mit Blick auf Bildung und Wissenschaft durch die allgemeine Anerkennung von Open-(Access)-Prinzipien. Open ist der Default nicht nur des Publizierens, sondern der Prozesse in Bildung und Wissenschaft allgemein.

Die dadurch angestoßene Transformation der Informationsmärkte ist längst noch nicht abgeschlossen und schon gar nicht hat die Gesetzgebung für das Urheberrecht dieser Transformation ausreichend Rechnung getragen. Ebenfalls ist es nicht sicher, ob durch diese Transformation der kommerziellen Verwertung auch von öffentlich produziertem Wissen Einhalt geboten werden kann. Ob als Dauerzustand oder nur als Übergang - derzeit zeigt sich die Öffentlichkeit durchaus bereit, die kommerzielle Wirtschaft auch im allgemeinen Open-Access-Paradigma auf den wissenschaftlichen Informationsmärkten nicht nur am Leben zu halten,

639 Vgl. (Kuhlen 2004e) Nachhaltigkeit muss nicht Verknappung bedeuten - in Richtung Wissensökologie; (Kuhlen 2004f) Artikel Wissensökologie und (Kuhlen 2012b) Wissensökonomie und Wissensökologie zusammen denken.

640 Die Leitidee der Nutzungsfreiheit sollte keine Privilegierung von Bildung und Wissenschaft bedeuten - obgleich dies hier im Vordergrund stand -, sondern bezieht sich auch die Nutzung von Wissen in der Öffentlichkeit, also durch jedermann, für alle. Vgl. (Chan et al. 2019) Situating Open Science: "Contextualizing Openness aims to stimulate further research and debates about how to collectively design a knowledge system that is open and equitable for all.”; ebenso Nick Shockey, posted 25.10.2019 on International Open Access Week: For Whom? Prompting Ourselves to Center Equity Year-Round - https://bit.ly/2pjephH; vgl. das Plädoyer von Klaus Graf für eine Öffnung der elektronischen Ressourcen der Bibliotheken über einen für jedermann erwerblichen Bibliotheksausweis. Remote Access und Open Access. Archivalia 27.10.2019 - https://bit.ly/2BVSGib. 
sondern auch als weiter bestimmenden „Partner“ anzuerkennen - mit der Konsequenz, dass auch die Open-Access-Märkte entscheidend von den kommerziellen Informationsmärken dominiert werden. Die Bereitschaft von öffentlich finanzierten Einrichtungen, für einen in einer (kommerziellen) Open-Access-Zeitschrift zu erscheinenden Artikel eine Publikationsgebühr zu zahlen - das geschah lange Zeit über eine APC -, lässt deutlich erkennen, dass die Leistung der kommerziellen Verwerter nicht nur anerkannt wird, sondern dass diese Leistung durch finanzielle Unterstützung stabil gehalten werden soll. Einen Schritt noch weiter gehen Vereinbarungen wie der Deal im Rahmen von DEAL (ausführlich dazu in 14.8). Trotzdem, auch wenn einiges darauf hindeutet, dass die Verlagskonsortien sich bislang als Gewinner des deal sehen können, ist DEAL doch ein wichtiger Schritt in die vollständige Transformation der Publikationsmärkte in Open-AccessMärkte mit dem neuen Paradigma der Nutzungsfreiheit - zumal dann, wenn die DEAL-Vereinbarungen nur als Übergangslösung eingeschätzt würden.

Wenn es Dauerlösungen sein sollten, kann aber durchaus die Frage gestellt werden, ob diese Transformation nicht besser und auf Dauer auch kostengünstiger erreicht werden könnte, wenn die umfänglichen, den Verlagen bereitgestellten finanziellen Mittel für die Entwicklung von Open-Access-Produkten und Dienstleistungen aus der Wissenschaft selbst eingesetzt würden. Wissenschaftler bzw. die ihnen zugeordneten Organisationen (wie z. B. Bibliotheken, Fachgesellschaften) sind heute technisch und organisatorisch in der Lage - und es gibt auch genug Beispiele dafür, dass sie es tun -, das Publizieren selbst in die Hand zu nehmen. Diese Möglichkeiten gelten nicht nur für das Publizieren von Forschungsergebnissen, sondern sie gelten auch für auf Lehre und Lernen bezogene Produkte. OER-Produkte sind realistische Alternativen zu den klassischen kommerziellen Lehrbüchern auch dadurch, dass sie auf Kollaboration und dynamische hypertextähnliche Weiterentwicklung setzen. ${ }^{641}$

Die Frage stellt sich tatsächlich, ob durch die öffentliche Finanzierung nicht das am Leben gehalten wird, was für sich nicht mehr marktfähig ist, sondern

641 (Kreutzer/Lahmann 2019) Rechtsfragen bei Open Science weisen darauf hin, dass auch mit den über freie Lizenzen (wie Creative Commons) zur Verfügung gestellten Open-Produkten durchaus Geld verdient werden kann. Allerdings darf dann kein Entgelt für die Nutzung selbst verlangt werden. Als Beispiel führen sie an, dass auch OER-Repositories kostenpflichtig angeboten werden können: „Zugriffsgebühren für einen Online-Dienst sind keine Lizenzgebühren, sie werden für die Nutzung des Dienstes gezahlt und nicht für die Nutzungsrechte an den Inhalten, die dort verfügbar sind. OER-Repositorien können daher kostenpflichtig angeboten werden, ohne dass dies den Prinzipien von OER oder gar den hierbei eingesetzten offenen Lizenzen widersprechen würde.“ Die Autoren stellen aber durchaus in Frage, ob so etwas „sinnvoll oder ratsam“ sei. Vor allem bei öffentlich finanzierten Organisationen wie Bibliotheken etc. sollte diese Frage wohl verneint werden. 
quasi auf öffentliche „Subventionen“ angewiesen ist. Vielleicht waren die wissenschaftlichen Informationsmärkte nie im klassischen Sinne Märkte - immer waren die öffentlich finanzierten Bibliotheken die gesicherten Hauptabnehmer der Produkte -, aber lange gab es dazu keine Alternativen. Diese wären heute gegeben. Wahrscheinlich wird es auch in Zukunft weiter kommerzielle Produkte und Dienstleistungen auf den Informationsmärkten geben. Diese werden sich aber abnehmend auf die Umwandlung der von den Urhebern verfassten Wissensobjekte in veröffentlichungs- und nutzungsfähige Informationsobjekte, also auf das bloße Publizieren beziehen. Ob neue kommerzielle Produkte mit informationellen Mehrwertleistungen und Wissenschaft unterstützende Dienstleistungen, wie sie sich derzeit über Konzepte wie Research Intelligence Provider (REP) von Elsevier abzeichnen (vgl. 14.8), über das Urheberrecht geschützt werden sollen/müssen, ist eine eher negativ zu beantwortende Frage. Entsprechend der bisherigen Systematik könnte das möglicherweise über spezielle Leistungsschutzrechte geregelt werden.

\section{Konsequenzen für das Urheberrecht}

Wissenschaft entwickelt sich schneller als das Recht in der Lage ist, auf diese Entwicklungen zu reagieren. Fast systematisch zwingend ist Wissenschaft immer mit den aktuell geltenden rechtlichen Vorschriften für den Umgang mit Wissen und Information unzufrieden. Eine Weile kann Wissenschaft noch mit unzulänglichen rechtlichen Regelungen leben, sei es durch schlichtes Ignorieren der bestehenden Vorschriften - in der bislang berechtigten Erwartung, dass im Wissenschaftsbereich kein Rechtsinhaber Verstöße gegen bestehende Urheberrechtsregelungen vor Gericht bringen wird - oder sei es durch erfindungsreiche Ersatzlösungen, durch die, als Voraussetzung für Wissenschaftsfreiheit, die freie Einsicht und freie Nutzung des öffentlich gemachten Wissens gesichert wird. Soll das Urheberrecht dabei eine wichtige Rolle spielen, wäre dafür eine Bezeichnung wie „Nutzungsrechte und Nutzungsfreiheiten für Wissen und Information“ angemessener als „Urheberrecht“. Um kein Missverständnis aufkommen zu lassen - mit „Nutzungsrechten“ sind nicht in erster Linie die Nutzungsrechte von Verlagen gemeint, durch die sie die Wissensobjekte der Urheber zur Verwertung nutzen zu dürfen. „Nutzungsrechte“ haben in dem Vorschlag „Nutzungsrechte und Informationsfreiheiten für Wissen und Information“ eine fünffache Bedeutung:

- Nutzungsrechte beziehen sich zunächst auf Autoren selbst. Diese nutzen ihr Wissen für Veröffentlichungen und haben das Recht zu entscheiden, ob, wann und wie sie ihr erarbeitetes Wissen öffentlich machen wollen.

- Nutzungsrechte sind auch das Recht von Personen, das Wissen Anderer frei zu nutzen - vor allem um in der Wissenschaft selbst dadurch Autor werden zu können. 
- Nutzungsrechte sind auch das institutionelle Nutzungsrecht der die Wissenschaftler tragenden, finanzierenden und bedienenden Organisationen.

- Nutzungsrechte sind auch das Recht der Öffentlichkeit an der freien Nutzung der veröffentlichten und i.d. R. auch von ihr finanzierten Werke:

- Schließlich sind Nutzungsrechte auch auf Verleger bezogen als das zu erwerbende Recht auf kommerzielle Verwertung der von Autoren produzierten Wissensobjekte.

Die hier vorgeschlagene Bezeichnung „Nutzungsrechte und Nutzungsfreiheiten für Wissen und Information“ könnte in einer Präambel des Urheberrechts - §1 ersetzend oder erweiternd - verwendet werden. In einem solchen Recht gelten weiter die durch das jetzige Urheberrecht uneingeschränkt geschützten und nicht durch Dritte in Frage zu stellenden Persönlichkeitsrechte. ${ }^{642}$ Aber angesichts der in allen Disziplinen, aber besonders in den STM-Fächern erkennbaren Tendenz zum kollaborativen Arbeiten in der Wissenschaft ist es wenig sinnvoll, exklusiv an dem individuellen Autor festzuhalten. Niemand aber kann und niemand will Autoren/Autorengruppen das Recht nehmen zu entscheiden, ob (und damit wann) und in welcher Form sie ihre erstellten Werke publizieren wollen. Das einklagbare Recht auf Anerkennung und Nennung der Autor-/Urheberschaft und der Schutz vor Entstellungen werden gerade in elektronischen Umgebungen keineswegs unwichtiger.

Anders sieht es bei den im Urheberrecht vorgesehenen Verwertungsrechten aus. Diese bislang exklusiv den Urhebern zustehenden Rechte werden durch das Open-Access-Paradigma bzw. durch die Leitidee der Nutzungsfreiheit überflüssig bzw. gegenstandslos. Mit einem Open Access gestellten Werk kann jeder weltweit genehmigungs- und vergütungsfrei das tun, was ursprünglich als Verwertungsrechte den Autoren exklusiv zustand, z. B. vervielfältigen, verbreiten, öffentlich zugänglich machen. ${ }^{643}$ Dies konsequent zu Ende gedacht, könnte das Wissenschaftsurheberrecht bzw. das Recht „Nutzungsrechte und Nutzungsfreiheiten für Wissen und Information“ im Open-Paradigma weitgehend auf die Verwertungsrechte und Teile des Urhebervertragsrechts verzichten. Dadurch würden tatsächlich all die bisherigen Schrankenregelungen mit ihren kleinteiligen Nutzungsvorschriften überflüssig. Entscheidend für die Nutzung sollte alleine der Zweck der in Bildung und Wissenschaft vorzunehmenden Handlungen sein. Dem kann ganz knapp,

$642 \mathrm{Ob}$ es möglich und sinnvoll ist, wie es in der Creative-Commons CCO-Lizenz vorgesehen ist, nämlich dass Autoren, die ihr Werk gänzlich in die Public domain stellen wollen, auf alle ihre Rechte, also auch auf ihre Persönlichkeitsrechte von sich verzichten können, kann hier nur als Frage gestellt werden.

643 Vgl. (Sandberger 2017) Die Zukunft wissenschaftlichen Publizierens. 
ohne Schranken, z. B. über die hier vorgeschlagene ABWK entsprochen werden. Die von Thomas Dreier 2019 gestellte Frage (Dreier 2019b), ob Schranken mit Blick auf Wissenschaft überhaupt nötig sind, wird hier, anders als von ihm, verneint.

Vielleicht müssen Übergangslösungen erhalten bleiben, weil der Großteil der weltweit (analog oder elektronisch) verfügbaren Artikel und Bücher früher rein kommerziell produziert wurde und diese entsprechend über die bisherigen Urheberrechtsregelungen geschützt sind. Auch wenn deren Bedeutung für viele wissenschaftliche Fächer abnehmen wird, sind diese doch Teil des kulturellen Erbes, das zugriffs- und nutzungsfähig gehalten werden muss. Ob das weiter durch das Urheberrecht geregelt werden muss oder durch eine Erweiterung von Verträgen wie DEAL, welche die Finanzierung der Erstellung von Informationsobjekten an die Freistellung des „Lesens“ dieser Objekte bindet, muss sich zeigen.

Zuweilen braucht es Revolutionen, um ganz neue politische Realitäten zu schaffen. Kopernikus hatte die Umlaufbahnen der Planeten um die Sonne in seinem Werk „De revolutionibus orbium coelestium“ (1543) beschrieben: Nicht mehr dreht sich die Sonne um die Erde, sondern die Erde um die Sonne. Das war dann tatsächlich eine Revolution, die unser Weltbild änderte. Revolutionen im Urheberrecht sind eher unwahrscheinlich. Vielleicht ist die Metapher der Kopernikanische Wende passender. Darunter wird, mit Kant, jede grundsätzlich andere Sicht auf bislang als unumstößlich angesehene Beschreibungen von Objekten und Sachverhalten in der Welt verstanden - so heute auch für eine grundlegende Reform des Urheberrechts. ${ }^{644}$ Julia Reda, Grüne Politikerin im EU-Parlament bis 2018, übernahm die Metapher der „Kopernikanischen Wende“, als sich (2015) über ihren umfassenden Vorschlag ${ }^{645}$ zumindest noch die Chance zu eröffnen schien, ein neues zeitgemäßes Urheberrecht durch eine neue EU-Richtlinie zu entwickeln.

Vorbilder für solche Wenden hat es aus der jüngeren Vergangenheit durchaus auch aus der Politik gegeben. 2010 versuchte es Till Steffen, Justizminister für Bündnis 90/Die Grünen im Senat der Freien und Hansestadt Hamburg in Hamburg ab 2008 (und dann wieder ab 2015). Er legte gemeinsam mit Netzpolitikern aus den Grünen ein Diskussionspapier „für ein nutzerorientiertes Urheberrecht“ vor. ${ }^{646}$ Darin werden auch Überlegungen in der Dissertation von Gerd Hansen aufgegrif-

644 (Kuhlen 2015a) Kopernikanische Wende in der EU-Urheberrechtsdebatte?

645 (Reda 2019) EU-Urheberrechtsreform: Der Kampf war nicht umsonst.

646 (Steffen 2010) Diskussionspapier „Nutzerorientierte Ausrichtung des Urheberrechts“. Dieser Text konnte nicht mehr über das Web ausfindig gemacht werden. 2010 habe ich mich in netethics ausführlich mit diesem Diskussionspapier auseinandergesetzt - https://bit.ly/2sBILgY. Die zentralen Formulierungen von Steffen, wie die hier im Text zitierten, sind dort umfänglich wiedergegeben; kritisch zu Steffens Vorschlägen: (Mönikes 2010) Anmerkungen zum Diskussionspapier „Nutzerorientierte Ausrichtung des Urheberrechts“ - https://bit.ly/2m36K4S. 
fen, ${ }^{647}$ in der dieser die These vertrat, dass das Urheberrecht nur dann akzeptabel bleiben kann, wenn es den Nutzerschutz stärker berücksichtigt. Hier einige Zitate aus diesem Steffen Papier:

\begin{abstract}
Das Urheberrecht, so wie es jetzt aussieht, passt nicht mehr zu den technischen Möglichkeiten elektronischen Umgebungen und der darin entwickelten Nutzungsgewohnheiten. [...] Das individualistische Begründungsmodell des Urheberrechts ist überholt. [...] Im Internet entstehen kreative Nutzergewohnheiten und Formate, die es schwierig machen, auch bei unterstelltem guten Willen fremde Urheberrechte zu beachten. [...] In der bisherigen Entwicklung des Urheberrechts sind diese mit der digitalen Entwicklung neu entstandenen Nutzungserwartungen weitgehend unberücksichtigt geblieben. [...] Die Rechtspolitik ist aufgerufen, den „Schrankenbegünstigten“ eine Rechtsposition zu verschaffen, die der Grundrechtsrelevanz der Schranken Rechnung trägt und die dem Recht des Urhebers ein Recht auf Nutzung an die Seite stellt.
\end{abstract}

Schließlich wird im Diskussionspapier von Steffen am Ende vorgeschlagen, im UrhG den Titel von $\S 1$ „Allgemeines“ durch „Zweck des Gesetzes“ zu ersetzen. Damit käme ein bislang im deutschen Urheberrecht eher vernachlässigtes utilitaristisches und damit die institutionellen Rechte unterstützendes Moment ins Spiel. Weiter sollte der bisherigen Formulierung in $\S 11$ UrhG, durch die bislang nur der Urheber (Satz 1) und dessen Recht auf Vergütung (Satz 2) geschützt wird, durch einen Satz ergänzt werden: „Zugleich trägt es den Bedürfnissen der Werknutzenden an der Teilnahme am kulturellen und geistigen Leben Rechnung. “ Dann sollte, so Steffen, dann gleich der gesamte Name des Gesetzes geändert werden in „Gesetz über Urheberrechte, verwandte Schutzrechte und Nutzungsfreiheiten (UrhG)“. Das erinnert schon sehr an den hier vorgeschlagenen Titel „Nutzungsrechte und Nutzungsfreiheiten für Wissen und Information.

Mittelfristig, aber ganz sicher langfristig kann sich das Recht nicht gegen sich entwickelnde Leitideen mit neuen Werten und neuen Verhaltensformen für Wissen und Information behaupten. Das macht die anfänglich vertretene These der Priorität von Ethik, Informationsethik, gegenüber den anderen Regulierungsinstanzen wie Technologien, Markt aber auch Recht aus. Die Priorität legitimiert sich dadurch, dass das Verhalten der Akteure für den Umgang mit Wissen und Information sich an normativ begründete Leitideen orientiert. Ein anderes Wort für „Verhalten“ ist in der Sprache der Commons das Commoning. Commoning ist die Verständigung der betroffenen Akteure (hier in Bildung und Wissenschaft) zum einen auf die den Leitideen zugrundeliegenden Werte wie Informationsautonomie, Nutzungsfreiheit, Gemeinwohl, Gerechtigkeit, Nachhaltigkeit und zum anderen auf Regeln, auch Sanktionen gegen Verstöße und Verhaltensformen wie Teilen und Kollaboration.

647 (Hansen 2009) Warum Urheberrecht? 
Die Ausführungsbestimmungen der Open-Access-Erklärungen sind Hinweise auf solches Commoning.

Die Prioritätsthese zugunsten der Ethik hat nichts mit moralisierender Politik zu tun. Priorität, bedeutet nicht, dass, entsprechend Platons erstem Vorschlag für eine gerechte Politik, die (informationsethischen) Philosophen „Könige“ werden sollen, aber vielleicht doch im Sinne seines zweiten Vorschlags, dass die „Könige“ sich informationsethisches Denken zu eigen machen bzw. die sich entwickelnde Leitidee des freien Umgangs mit Wissen und Information als handlungsrelevant annehmen und dies in die Sprache und Formalitäten des Rechts umsetzen. Kurzfristige „Siege“ der Informationsethik sind nicht zu erwarten. Aber ein Recht für Nutzungsrechte und Nutzungsfreiheiten sollte sich realisieren lassen.

Eine Utopie - so wird es Alphonse de Lamartine zugeschrieben - ist eine Idee, deren Zeit noch nicht gekommen ist. Aber Nutzungsfreiheit in der Wissenschaft ist keine Utopie, sondern ist in der Zeit angekommen, und nichts - so wird es Victor Hugo zugeschrieben - ist so stark wie eine Idee, deren Zeit gekommen ist. 
